\title{
Fast Crown Scheduling Heuristics for Energy-Efficient Mapping and Scaling of Moldable Streaming Tasks on Manycore Systems
}

\author{
NICOLAS MELOT and CHRISTOPH KESSLER, Linköping University \\ JÖRG KELLER and PATRICK EITSCHBERGER, FernUniversität in Hagen
}

Exploiting effectively massively parallel architectures is a major challenge that stream programming can help facilitate. We investigate the problem of generating energy-optimal code for a collection of streaming tasks that include parallelizable or moldable tasks on a generic manycore processor with dynamic discrete frequency scaling. Streaming task collections differ from classical task sets in that all tasks are running concurrently, so that cores typically run several tasks that are scheduled round-robin at user level in a data-driven way. A stream of data flows through the tasks and intermediate results may be forwarded to other tasks, as in a pipelined task graph. In this article, we consider crown scheduling, a novel technique for the combined optimization of resource allocation, mapping, and discrete voltage/frequency scaling for moldable streaming task collections in order to optimize energy efficiency given a throughput constraint. We first present optimal offline algorithms for separate and integrated crown scheduling based on integer linear programming (ILP). We make no restricting assumption about speedup behavior. We introduce the fast heuristic Longest Task, Lowest Group (LTLG) as a generalization of the Longest Processing Time (LPT) algorithm to achieve a load-balanced mapping of parallel tasks, and the Height heuristic for crown frequency scaling. We use them in feedback loop heuristics based on binary search and simulated annealing to optimize crown allocation.

Our experimental evaluation of the ILP models for a generic manycore architecture shows that at least for small and medium-sized streaming task collections even the integrated variant of crown scheduling can be solved to optimality by a state-of-the-art ILP solver within a few seconds. Our heuristics produce makespan and energy consumption close to optimality within the limits of the phase-separated crown scheduling technique and the crown structure. Their optimization time is longer than the one of other algorithms we test, but our heuristics consistently produce better solutions.

Categories and Subject Descriptors: C.2.2 [Multicore and GPU Programming]: Parallel Programming

General Terms: Design, Algorithms, Performance

Additional Key Words and Phrases: Multicore, manycore, parallel energy, scheduling, mapping, frequency scaling, streaming

\section{ACM Reference Format:}

Nicolas Melot, Christoph Kessler, Jörg Keller, and Patrick Eitschberger. 2014. Fast crown scheduling heuristics for energy-efficient mapping and scaling of moldable streaming tasks on manycore systems. ACM Trans. Architec. Code Optim. 11, 4, Article 62 (December 2014), 24 pages.

DOI: http://dx.doi.org/10.1145/2687653

C. Kessler acknowledges partial funding by EU FP7 EXCESS, Vetenskapsrådet and SeRC. N. Melot acknowledges partial funding by the CUGS graduate school at Linköping University.

Authors' addresses: N. Melot and C. Kessler, Linköping University, Department of Computer and Information Science (IDA), S-58183 Linköping, Sweden; emails: \{nicolas.melot, christoph.kessler\}@liu.se; J. Keller and P. Eitschberger, FernUniversität in Hagen, Faculty of Mathematics and Computer Science, 58084 Hagen, Germany; email: \{joerg.keller, patrick.eitschberger\}@fernuni-hagen.de.

Permission to make digital or hard copies of part or all of this work for personal or classroom use is granted without fee provided that copies are not made or distributed for profit or commercial advantage and that copies show this notice on the first page or initial screen of a display along with the full citation. Copyrights for components of this work owned by others than ACM must be honored. Abstracting with credit is permitted. To copy otherwise, to republish, to post on servers, to redistribute to lists, or to use any component of this work in other works requires prior specific permission and/or a fee. Permissions may be requested from Publications Dept., ACM, Inc., 2 Penn Plaza, Suite 701, New York, NY 10121-0701 USA, fax +1 (212) 869-0481, or permissions@acm.org.

(c) 2014 ACM 1544-3566/2014/12-ART62 $\$ 15.00$

DOI: http://dx.doi.org/10.1145/2687653 


\section{INTRODUCTION}

The increasing attention given to multi- and manycore architectures points to the need for programming models able to easily profit from their power. Among many solutions to the general parallel programming problem, the streaming approach is particularly adapted to coarse architectures and provides opportunities for the design of highly scalable parallel algorithms [Gordon et al. 2006; Lee and Messerschmitt 1987]. Examples include image processing and video encoding/decoding pipelines, as well as other applications operating on large data volumes, for example, streamed mergesort or mapreduce. We consider the steady state of a software pipelined streaming task application in which all streaming tasks are active simultaneously. Each task repeatedly consumes some amount of input, does some computation, and produces output that is forwarded to another task (or to memory if it is a final result).

A streaming task application can be modeled by a static streaming task graph (or a Kahn Process Network [Kahn 1974]) in which the nodes represent the tasks, which are not annotated with runtimes but with average computational rates. In the steady state, an edge between tasks $u$ and $v$ does not indicate a precedence constraint but denotes a communication of outputs from producer task $u$ to become inputs of consumer task $v$, and is annotated with an average bandwidth requirement.

As the workloads of different tasks may vary around the given averages and as there are normally more tasks than cores on the underlying machine, dynamic round-robin scheduling enables several tasks to run concurrently on the same core. In this case, a scheduling point is assumed to occur after the production of a packet, and a round-robin nonpreemptive user-level scheduler normally is sufficient to ensure that each task gets its share of processor time, provided the core has enough processing power, that is, it is run at a frequency high enough to handle the total load from all tasks mapped to this core.

Here we consider streaming task collections, that is, we model the tasks' computational loads only. Our results can still be applied to streaming task graphs if we assume that the latencies of producer-consumer task communications can be hidden by pipelining with multi-buffering from one scheduling round to the next, and that on-chip network links are not oversubscribed ${ }^{1}$.

We assume that our underlying machine consists of $p$ identical processors, which can be frequency scaled independently. We consider discrete frequency levels. We do not consider voltage scaling explicitly, but as most processors auto-scale the voltage to the minimum possible for a given frequency, this is covered as well. We allow for arbitrary core power models, which might comprise static and dynamic power consumption, and can be modeled analytically or based on measurements on real hardware. Beyond analytic power models like $f^{\alpha}$, other models (e.g., derived from measurements) are also possible by replacing the corresponding terms in integer linear programming (ILP) formulations and heuristics.

A task instance produces a packet of output data from packets of input data. Its granularity depends both on the data packet size and on the complexity of the computation performed on it. In practice, we assume task instances (i.e., per packet) to take a time in the milliseconds range. This is coarse enough that the per-task user-level scheduling overhead, buffer management and frequency switching overhead can be considered reasonably low compared to task execution time. We allow for moldable (multithreaded, or parallelizable) tasks that can internally use a parallel algorithm involving multiple processors (possibly using communication and synchronization via shared memory or message passing) in order to achieve parallel speedup. We do not schedule tasks in

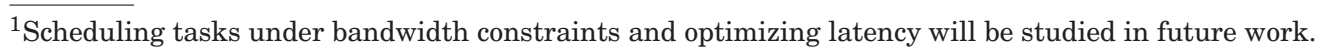


a malleable way, that is, a task cannot increase or decrease its number of allocated cores while running. Such task collections can still be scheduled by considering them as moldable tasks. We make no assumptions about the parallel efficiency functions of moldable tasks; these are parameters of our model. Moldable, partly moldable, and sequential tasks might be mixed.

We are interested in (1) statically allocating processing resources to tasks and (2) mapping the tasks to processors in such a way that (3) after suitable task-wise frequency scaling given a throughput constraint and overall energy consumption during one full round of the round-robin scheduler is minimized. We refer to this combined optimization problem (1-3) shortly as energy-efficient scheduling. With this respect, we make the following contributions:

-We introduce the crown structure as a constraint on resource allocation, which reduces the complexity of allocation, mapping, and discrete frequency scaling considerably, namely, from $p$ to $O(\log p)$ possible task sizes for allocation and from $2^{p}$ to $O(p)$ possible processor subsets for mapping. We show that this constraint makes the exact solution of the still considered NP-hard optimization problem feasible even for medium problem sizes. We argue that the resulting schedules are efficient and also flexible for dynamic rescaling to adapt to fluctuations in streaming task load at runtime, in order to save further energy, for example, in situations in which a task may not be data-ready in a schedule round and has to be skipped. We present crown scheduling, a novel method for energy-efficiency scheduling under the crown structure constraint.

-We show how to apply discrete frequency scaling of the tasks in a given crown schedule to optimize its energy efficiency for a given throughput constraint.

-We give implementations of crown scheduling as ILP models and evaluate them for a generic manycore architecture, showing that it can be solved to optimality (with regard to the crown structure) for small and most medium-sized streaming task collections within a few seconds.

-We present efficient heuristics for crown allocation, mapping and discrete frequency scaling phases for large streaming task collections. We give a time complexity analysis for each of them and show that the quality of their solution competes with optimal crown schedules for a much lower optimization time.

The remainder of this article is organized as follows: Section 2 introduces the general concepts and central notation. Section 3 considers the separate optimization of crown resource allocation/mapping and subsequent frequency/voltage scaling, while Section 4 provides the integrated solution of crown resource allocation, mapping and scaling in the form of an ILP model that can provide an optimal solution for small and mediumsized problems. Section 5 addresses dynamic schedule rescaling. Section 6 presents experimental results. Section 7 discusses related work. Section 8 presents conclusions and proposes future work.

\section{CROWN SCHEDULING}

A crown ${ }^{2}$ is a recursive $b$-ary decomposition of the set of cores into subgroups down to singletons. These subgroups are to become the only possible mapping target for tasks. The base of a crown represents the full core set to run very parallel tasks. This interdependency decreases with the parallel degree of tasks and the increasing height in the crown. The crown structure aims to guarantee that cores belonging to a higher region of the crown (running less parallel tasks) can perform their work independently of cores belonging to another branch.

\footnotetext{
${ }^{2}$ The name is inspired by the shape of a crown, whose triangular spikes symbolize a decreasing degree of connectivity; see Figure 1(a).
} 
We consider a generic multicore architecture with $p$ identical cores (processors). For simplicity of presentation, we assume in the following that $p=2^{L \text { (see } 3)}$ is a power of $2^{\text {(see } 4)}$. All $p$ cores can be individually and dynamically set to run at one frequency level from a finite set $F$.

The set of processors $P=\left\{P_{1}, \ldots, P_{p}\right\}$ is hierarchically structured into $2 p-1$ processor subgroups by recursive binary partitioning as follows: The root group $G_{1}$ equals $P$; it has the two child subgroups $G_{2}=\left\{P_{1}, \ldots, P_{p / 2}\right\}$ and $G_{3}=\left\{P_{p / 2+1}, \ldots, P_{p}\right\}$, four grandchildren groups $G_{4}=\left\{P_{1}, \ldots, P_{p / 4}\right\}$ to $G_{7}=\left\{P_{3 p / 4+1}, \ldots, P_{p}\right\}$ and so on over all $L+1$ tree levels, up to the leaf groups $G_{p}=\left\{P_{1}\right\}, \ldots, G_{2 p-1}=\left\{P_{p}\right\}$. Unless otherwise constrained, such grouping should also reflect the sharing of hardware resources across processors, such as on-chip memory shared by processor subgroups. Let $C_{m}$ denote the set of all groups that contain processor $P_{m}$. For instance, $C_{1}=\left\{G_{2^{z}}: z=0, \ldots, L\right\}$. Let $p_{i}=\left|G_{i}\right|$ denote the number of processors in processor group $G_{i}$. Where it is clear from the context, we also write $i$ for $G_{i}$ for brevity.

We consider a set $T=\left\{t_{1}, \ldots, t_{n}\right\}$ of $n$ moldable, partly moldable or sequential streaming tasks, where each task $t_{j}$ performs work $\tau_{j}$ and has a maximum width $W_{j} \geq 1$ and an efficiency function $e_{j}(q)>0$ for $1 \leq q \leq p$ that predicts the parallel efficiency (i.e., parallel speedup over $q$ ) with $q$ processors. For moldable tasks, $W_{j}$ is $\infty$, that is, unbounded; for partly moldable tasks, $W_{j}$ can be any fixed value $>1$, and for sequential tasks $W_{j}=1$. For all tasks $t_{j}$ we assume that $e_{j}(1)=1$, that is, no parallelism overhead when running on a single processor ${ }^{5}$. Where clear from the context, we also write $j$ as shorthand for $t_{j}$.

Resource allocation assigns each task $t_{j}$ a width $w_{j}$ with $1 \leq w_{j} \leq \min \left(W_{j}, p\right)$, for $1 \leq j \leq n$. As additional constraint, we require for crown allocation that $w_{j}$ be a power of 2 , thus could be mapped completely to one of the $2 p-1$ processor subgroups introduced earlier. A crown mapping is a mapping of each task $t_{j}$ with assigned width $w_{j}$ to one of the processor subgroups in $\left\{G_{1}, \ldots, G_{2 p-1}\right\}$ of a size matching $w_{j}$. For each processor group $G_{i}, 1 \leq i \leq 2 p-1$, let $T^{(i)}$ denote the set of tasks $t_{j} \in T$ that are mapped to $G_{i}$. For each processor $P_{m}, 1 \leq m \leq p$, let $T_{m}$ denote the set of tasks $t_{j} \in T$ mapped to $P_{m}$, that is, to any $G_{i} \in C_{m}$. Conversely, let $R_{j}$ denote the group to which task $t_{j}$ is mapped. Crown scaling attributes a running frequency level to each task. If task $j$ is given frequency level $f_{j} \in F$, and mapped to group $i$, then all processors $G_{i}$ must run at frequency $f_{j}$ from when task $j$ begins until task $j$ finishes.

We define the execution time for tasks, groups, and processors as functions of allocation, mapping, and frequency settings given to all tasks. The execution time of a task $j$ running on $w$ processors at frequency $f$ is defined as follows:

$$
t t_{j}(w, f)=\frac{\tau_{j}}{f \cdot e_{j}(w) \cdot w}
$$

Where clear from the context, we write $t t_{j}$ for the execution time of task $j$ with given width $w_{j}$ and frequency $f_{j}$. We conveniently assume here that the running time of task $j$ is proportional to the frequency inverse $1 / f$, but the model allows extensions to sample the tasks' speedup function of frequency. Similarly, we consider the fact

\footnotetext{
${ }^{3}$ We can define $p=b^{L}$ with $b>1$ and $b \in \mathbb{N}$ for generality. However, our method yields more optimization opportunities as $b$ decreases, for the same complexity. Therefore, in this article, we are only interested in the case in which $b=2$.

${ }^{4}$ A generalization towards (nonprime) $p$ that are not powers of 2 , such as $p=48$ for Intel SCC, derives from a recursive decomposition of $p$ into its prime factors a corresponding multidegree tree structure instead of the binary tree structure described in the following for organizing processors in processor groups.

${ }^{5}$ In principle, partial moldability might also be expressed by manipulating the $e_{j}$ functions, but in this article we use the upper limits $W_{j}$ for this purpose. We may assume that the efficiency functions $e_{j}$ are monotonically decreasing, although this assumption is not strictly necessary for the techniques of this article.
} 
that tasks use all $w$ through all their entire execution time ${ }^{6}$. The static runtime of a processor group $i$, denoted $g t_{i}$, is the sum of execution times of all tasks mapped to this group. If they all run at frequency $f$, then we write $g t_{i}(f)$ as:

$$
g t_{i}(f)=\sum_{j \in T^{(i)}} t t_{j}\left(w_{j}, f\right)
$$

We write for convenience $g t_{i}=\sum_{j \in T^{(i)}} t t_{j}\left(w_{j}, f_{j}\right)$ for the execution time of group $i$, with all tasks $j$ running at the frequency $f_{j}$ that they have been assigned. A processor $m$ runs as long as the sum of all execution times of all groups it belongs to, assuming frequency $f$ :

$$
p t_{m}(f)=\sum_{i \in C_{m}} g t_{i}(f) .
$$

When clear from the context, we also write $p t_{m}=\sum_{i \in C_{m}} g t_{i}$ for the execution time of processor $m$, where all tasks $j$ that it executes run at the frequency $f_{j}$ that they are assigned. When we need to compare task $j$, group $i$, or processor $m$ by unscaled execution time, that is, with no bias due to any task frequency settings, for instance, to compute the task's running frequency, we use the constant frequency $1 \mathrm{~Hz}$ with $t t_{j}\left(w_{j}, 1\right)$ (Equation (1)), gt $t_{i}(1)$ (Equation (2)) and $p t_{m}(1)$ (Equation (3)), respectively.

For each processor $P_{m}$, we order the tasks in $T_{m}$ in nonincreasing order of width, for example, by concatenating the elements of all $T^{(i)} \in C_{m}$ in increasing order of group index $i$. The relative order of the tasks within each processor group must be kept the same across all its processors, for example, in increasing task index order. We call such a $p$-tuple of processor schedules a crown schedule.

A crown scheduler is a user-level round-robin scheduler running on each processor $P_{m}, 1 \leq m \leq p$, that uses a crown schedule for the (instances of) tasks in $T_{m}$ to determine their order of execution. A crown scheduler works in rounds. Each round starts with a conceptual global barrier synchronization across all $p$ processors ${ }^{7}$. Thereafter the tasks are executed round-robin as specified in the chosen crown schedule of the tasks in $T_{m}$. Tasks that are not data ready are skipped. Then the next round follows. The stable state of a round in a crown-based streaming computation involves (almost) all tasks in $T$ as they all have data to process. See Figure 1(b) for an illustration.

The processing of tasks in decreasing order of width is required to make sure that all processors participating in the same moldable task (instance) start and stop approximately at the same time, allowing for efficient interprocessor communications and avoiding idle times, except at the end of a round. Between tasks (even of different widths) within a round, scheduled idle time ("external fragmentation") cannot occur because the processors of the subgroup assigned to the subsequent task automatically start quasi-simultaneously after finishing the previous task in which they also participated; a moldable task is either data ready or not, which means it is skipped either by all or none of the processors in the assigned processor group.

Crown scheduling computes crown allocation, mapping, and frequency scaling for streaming tasks and minimizes energy consumption under a makespan constraint. We fix a target throughput for an application as the inverse of a time budget $M$ to execute a

\footnotetext{
${ }^{6}$ If not, then task $j$ 's efficiency function but instead derive the parallel time function $t t_{j}$ of each task $j$ from analysis or measurements.

${ }^{7}$ There is no need for an explicit barrier to separate rounds. For the prediction of makespan, a conceptual barrier can be assumed: As the cores involved in a parallel task (e.g., the first full-width parallel task running on the root group at the beginning of a round) will task-internally communicate and synchronize with each other, the resulting waiting times that would occur if cores started the task at different times can conceptually be moved to the end of the preceding round, thus having the same effect as a zero-latency barrier separating the rounds.
} 


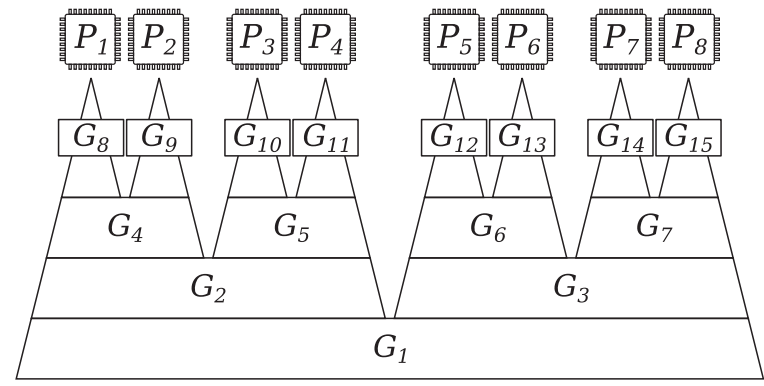

(a) The 15 processor groups in a binary crown over 8 processors, and their corresponding processors above.

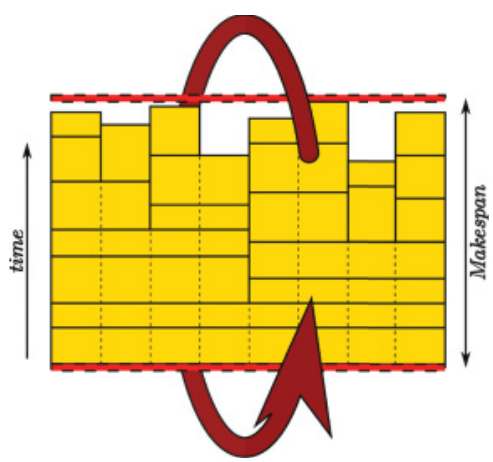

(b) An (unscaled) crown schedule running in round-robin fashion.

Fig. 1. A group hierarchy and possible corresponding unscaled crown schedule for eight processors.

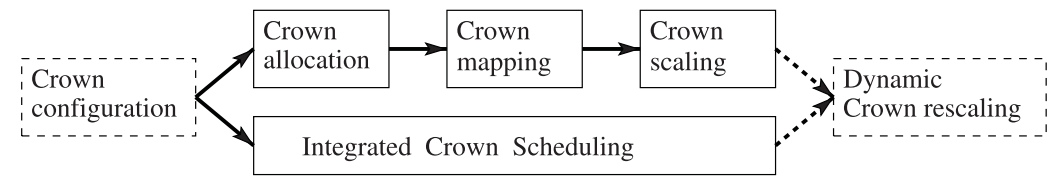

Fig. 2. Overview of phase-separated vs. integrated crown scheduling. The methods shown by solid boxes are detailed in this article.

round. Crown scheduling allows any analytic energy consumption model. Let $P C(f)$ be an arbitrary dynamic power function for the crown scheduler. For a concrete machine, it is sufficient to use results from power measurements of the target machine at the available frequencies [Cichowski et al. 2012]. In our experiments, we set $P C(f)=f^{\alpha}$ with $\alpha=3$. We consider the energy spent at idle time as negligible, and also ignore the time and energy required to switch frequencies, as they are small against the time of a round. Equation (4) shows our energy consumption model:

$$
\text { energy }=\sum_{j=1}^{n} t t_{j} \cdot w_{j} \cdot P C\left(f_{j}\right) \text {. }
$$

\section{PHASE-SEPARATED, ENERGY-EFFICIENT CROWN SCHEDULING}

In this section, we discuss separate optimization algorithms for each of the subproblems crown resource allocation, crown mapping, and crown scaling. An integrated and more expensive approach is be discussed in Section 4. An overview of these problems is given in Figure 2.

We can reduce overall energy usage by scheduling tasks to run at a frequency as low and uniform as possible through a pipeline stage [Li 2008]. Also, early phases of the phase-separated approach are unaware of their influence on the efficiency of subsequent phases. However, the more idle time processors have between the target makespan and the execution of their last task, the lower the frequency that can run their task and the lower the energy that a pipeline stage consumes. Therefore, the allocation and mapping phases aim at optimizing for unscaled execution time and load balancing. The frequency-scaling phase scales frequencies as low as possible under the makespan constraint.

In this section, we call crown-optimal an optimal solution of the allocation, mapping, or frequency-scaling phases in a separate manner. Because they are solved separately, they do not participate in an optimal solution to the general scheduling problem studied in this article. 


\subsection{Crown-Optimal Crown Resource Allocation}

Let $Q_{z}$, where $z=0, \ldots, \log _{2} p$, be the set of indexes of all the tasks that are assigned width $w_{j}=2^{z}$. Then task $t_{j}\left(j \in Q_{z}\right)$ has unscaled runtime $\hat{\tau}_{j}=t t_{j}\left(w_{j}, 1\right)$, assuming that its processors run at fixed frequency 1 . If we assume as a simplification that all tasks with the same width $2^{z}$ can be distributed over the $p / 2^{z}$ processor groups in a balanced fashion, the runtime of these tasks on $p$ processors is

$$
R_{z}=\sum_{j \in Q_{z}} \frac{\hat{\tau_{j}}}{p / 2^{z}} .
$$

Optimal resource allocation chooses the $Q_{z}$ in a way to minimize

$$
\sum_{z=0}^{\log p} R_{z}
$$

that is, we minimize the total runtime assuming that the processors run at a fixed frequency and the tasks of one width can be distributed optimally. To do this by linear optimization, we introduce binary variables $v_{j, z}$ with $v_{j, z}=1$ if task $j$ gets width $w_{j}=2^{z}$. Then Equation (5) transforms to

$$
R_{z}=\sum_{j} \frac{\hat{\tau_{j}} v_{j, z}}{p / 2^{z}}
$$

and we need the constraint

$$
\forall j: \sum_{z} v_{j, z}=1
$$

It is simple to find an allocation that minimizes the total runtime, under the assumption that the tasks can be distributed optimally, that is, the total runtime is given by Equation (5). Equation (5) is minimized if all $\hat{\tau}_{j} v_{j, z} /\left(p / 2^{z}\right)$ are minimized. Therefore, we compute for each task $j$ the speedup $g_{j}(q)=e_{j}\left(2^{q}\right) \cdot 2^{q}$ for each $q \in[0 . . L]$ and select one $q$ that maximizes $g_{j}$, from all $L=\log p$ possible allocations. Since we have $n$ tasks, this takes at most $O(n \cdot \log p)$ time steps in total. Because all terms in the sum of Equation (5) are independent and because we minimize each, the computed allocation is optimal. Because it computes an allocation such that each task runs individually for the shortest possible time, we refer to this algorithm as fast allocation.

\subsection{Crown-Optimal Task Mapping}

For an optimal crown mapping given an allocation, we treat all widths separately, that is, we solve $\log _{2} p$ smaller optimization problems. For the tasks $j$ of a given width $w_{j}=2^{z}$, we try to distribute them over the $p / w_{j}$ processor groups available such that the maximum load over any processor is minimized.

In order to formulate the mapping of the tasks of a given width $2^{z}$ as an ILP, we minimize a variable maxload (note that the target function here is simple) under some constraints. To express the constraints, we use binary variables $y_{j, i}$ where $j$ runs (in consecutive numbering) over all tasks with width $2^{z}$ and $i$ runs over all considered $p / 2^{z}$ processor groups $G_{p / w_{j}}, \ldots, G_{2 p / w_{j}-1}$. Hence, $y_{j, i}=1$ if task $j$ is mapped to processor group $i$. The constraints are as follows. Task $j$ is mapped to exactly one processor group among those that have the right width $w_{j}$ :

$$
\forall j \text { with width } w_{j}=2^{z}: \sum_{i=p / w_{j}}^{2 p / w_{j}-1} y_{j, i}=1 .
$$




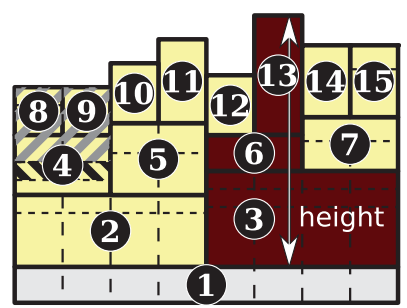

(a) Height of a schedule for eight processors and processor groups 3,6 , and 13 , and area (gray hash) to be rescaled if the back hashed task is delayed or skipped.

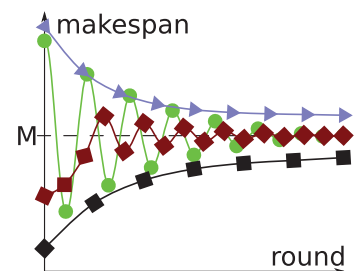

(b) Four binary search scenarios: (1) Minimal efficiency increase does not prevent valid schedules (bottom, plain black squares); (2) search for highest valid value from the beginning (mid-dark green dots); (3) minimal efficiency increase prevents valid schedules, then searches for higher valid value (dark-red diamonds); and (4) binary search fails to find any allocation (top, light blue triangles).

Fig. 3. Illustration of the Height of a schedule for eight processors and four possible scenarios of schedules' makespan evolution during binary search.

We define $T_{i}$ as the load mapped to processor $i$, that is, the sum of the load of each group $i$ is part of

$$
\forall i \in 1 . . p: T_{i}=\sum_{q \in C_{i}} \sum_{j=1}^{n} \hat{\tau}_{j} \cdot y_{j, q}
$$

and maxload as the maximal $T_{i}$, that is, the makespan:

$$
\operatorname{maxload} \geq \max _{i \in 1 . . p}\left(T_{i}\right) .
$$

\subsection{Heuristic Task Mapping with Load Balancing}

We describe the Longest Task, Lowest Group (LTLG) mapping heuristic for the load balancing problem modeled in Section 2. We consider the height of a processor group $i$ as shown in Equation (6) and illustrated in Figure 3(a). The height of a group $i$ is the maximum running time $\max _{m \in G_{i}} p t_{m}(f)$ of all its processors running at frequency $f$. We call for convenience height $t_{i}$ the height of group $i$ where all tasks $j$ run at frequency $f_{j}$ (Equation (7)). The LTLG heuristic discards all tasks of group 1 from the height of any group because group 1 cannot participate in any load imbalance (height $\left.t_{i=1}=0\right)$. As no frequency scaling is performed yet at the mapping stage (Figure 2), LTLG computes the height of a group $i$ for a constant frequency $c\left(\right.$ height $\left._{i}(c)\right)$. Note that this is not a limitation of our LTLG heuristic, as scaled tasks would only result in a different running time for each task to map.

$$
\begin{gathered}
\text { height }_{i \in[1,2 p-1]}(f)=g t_{i}(f)+\sum_{i^{\prime}=2}^{\left\lfloor\log _{2} i\right\rfloor} g t_{\left\lfloor\frac{i}{2^{i^{\prime}}}\right\rfloor}(f)+\max \left(\text { height }_{2 i}(f), \text { height }_{2 i+1}(f)\right) \\
\text { height }_{i \in[1,2 p-1]}=g t_{i}+\sum_{i^{\prime}=2}^{\left\lfloor\log _{2} i\right\rfloor} g t_{\left\lfloor\frac{i}{2^{i^{\prime}}}\right\rfloor}+\max \left(\text { height }_{2 i}, \text { height }_{2 i+1}\right)
\end{gathered}
$$

LTLG is based on three principles to produce better load-balanced mappings:

(1) The assignment of each task $j$ to the group $i$ of least height $t_{i}$ among groups of size $w_{j}$ limits load imbalance while building a schedule.

(2) The insertion of tasks of highest parallel running time first (with $w_{j}$ processors) lowers the risk of creating load imbalance when adding the last tasks to a schedule. 

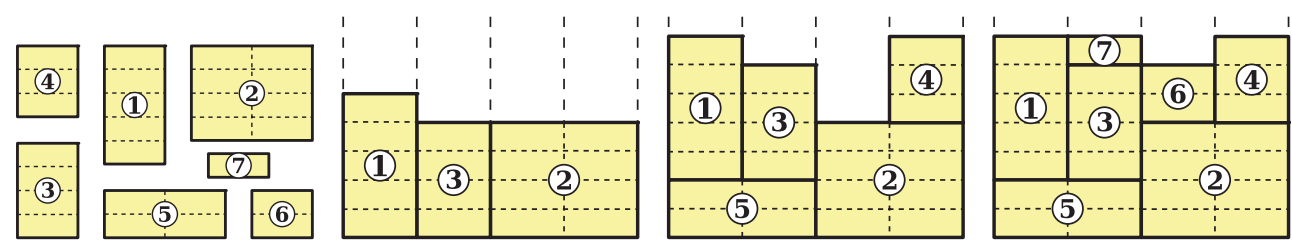

(a) Task set sorted and (b) Map tasks 1,2, and 3 to (c) Map task 4 to group 7 (d) Map tasks 6 and 7 to numbered by parallel run- groups 4,5 , and 3 , and start $\left(P_{4}\right)$ and task 5 to group 3 the sequential groups run ning time and width as of at $\mathrm{t}=0$ as no processor has $\left(P_{1}\right.$ and $\left.P_{2}\right)$, under tasks by the least loaded procesprinciples 2 and $3 . \quad$ any job scheduled yet. of lower parallel degree. sors.

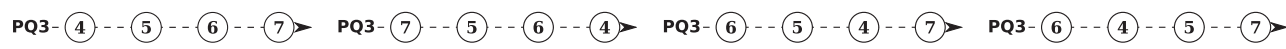

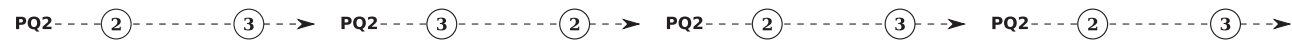

(e) Initial state of prior- (f) After inserting task 3. (g) Possible ity queues. The left-most Groups 4 and 2 must be queues after group in a level is the last of their queues as task 5. top priority. their height is the highest.

priority (h) Possibe final priority inserting queues. Groups 4, 5, 7, 2 and 3 could be ordered differently.

Fig. 4. Running example of 7 tasks mapped with LTLG yielding a makespan of 7. Tasks are sorted and numbered after their parallel running time and degree as described by principles 2 and 3. Tasks 1 to 7 with their current allocation run for $5,4,4,3,2,2$, and 1 time units. The reader may build schedules without principles 2 and 3 (or using their complement instead) to experience their contribution.

(3) If tasks have the same parallel running time, inserting tasks of highest width first keeps lowest width tasks available to later fill in the schedule's holes.

The algorithm maintains $\log _{2} p$ priority queues, one per group size. The priority of groups is defined by their least height, or their group number if both groups have the same current height (Eq. (8)). Since tasks of size $w_{j}=p$ do not create load imbalance and as the root group is the only group of size $p$, we do not use a priority queue for it. The algorithm first sorts the tasks using Equation (9) to compare them, with both their parallel running time and their width. Considering tasks in this order, we assign task $j$ to the highest priority group $i$ of size $w_{j}$ as given by the priority queue for size $w_{j}$. Then we recompute the runtime gt $t_{i}$ of group $i$ and its height, as well as for all its children groups recursively. If the new height of group $i$ is the maximum height among all children of group $i^{\prime}=\lfloor i / 2\rfloor$, then the height of $i^{\prime}$ is also updated, and this is repeated for $i^{\prime}$ until the root group (excluded) is reached.

$$
\begin{gathered}
\phi\left(i_{1}, i_{2}\right)= \begin{cases}\text { height }_{i_{1}}>\text { height }_{i_{2}} & \text { if height } i_{1} \neq \text { height }_{i_{2}} \\
i_{1}>i_{2} & \text { otherwise. }\end{cases} \\
\phi\left(j_{1}, j_{2}\right)= \begin{cases}t t_{j_{1}}\left(w_{j_{1}}, 1\right)>t t_{j_{2}}\left(w_{j_{2}}, 1\right) & \text { if } t t_{j_{1}}\left(w_{j_{1}}, 1\right) \neq t t_{j_{2}}\left(w_{j_{2}}, 1\right) \\
w_{j_{1}}>w_{j_{2}} & \text { if } t t_{j_{1}}\left(w_{j_{1}}, 1\right)=t t_{j_{2}}\left(w_{j_{2}}, 1\right) \text { and } w_{j_{1}} \neq w_{j_{2}} \\
j_{1}>j_{2} & \text { otherwise. }\end{cases}
\end{gathered}
$$

At each insertion of task $j$ to a group $i$, we recompute the group's runtime $g_{i}$. This takes constant time as we can add $\tau_{j}$ to an existing $g t_{i}$ value, initialized to 0 . Then, we need to update the height of all $O(p)$ descendant groups of $i$ as well as all $O(\log p)$ ancestor groups of $i$. Each group update requires a constant time update of the group starting time for descendant groups and of the maximum child height for ancestor groups. Finally, for each height recomputed, the group list for the corresponding level must be sorted to reflect the new height. This can take $O(\log p)$ time by removing and inserting again the group in the list. Each task insertion, therefore, takes $O(p \log p+$ $\log ^{2} p$ ) time steps. As we insert $n$ tasks, the time complexity of the LTLG heuristic is $O\left(n\left(p \log p+\log ^{2} p\right)\right)=O(n p \log p)$. 


\subsection{Crown-Optimal Voltage/Frequency Scaling of Schedules}

We assume that when running all cores at maximum speed $F_{\max }$ all the time, the resulting makespan is $\hat{M} \leq M$. The gap $M-\hat{M}$ and any processor-specific idle times at the "upper end" of the crown can then be leveraged for voltage/frequency scaling to obtain better energy efficiency. We call this "scaling the schedule."

For a given crown, energy efficiency can be optimized by each processor running each of its tasks $t_{j}$ requiring work $\tau_{j}$ at a frequency $f_{j}$ chosen from a given set $F=\left\{F_{1}, \ldots, F_{s}\right\}$ of $s$ discrete (voltage and) frequency levels, such that it still meets the throughput constraint. We require

$$
\forall m=1, \ldots, p: p t_{m} \leq M
$$

and the overall energy usage as defined by Equation (4) is minimized, subject to the additional constraint that a processor of a group must use the same frequency level for a task.

\subsection{Height Heuristic for Voltage/Frequency Scaling}

The Height frequency scaling heuristic reduces individual tasks' frequencies so that the final schedule satisfies the throughput constraint $M$ and tries to minimize energy consumption. In general, energy consumption is lower when all tasks run with the same frequency compared to running them at different frequencies for performing the same work in the same time interval [ $\mathrm{Li} 2008]$. Therefore, we decrease moderately the frequency of all tasks together instead of decreasing aggressively the frequency for some of the tasks while greatly increasing the frequency of other tasks in order to satisfy the makespan constraint $M$. Our heuristic is an implementation of the Best-Fit heuristic for the bin packing problem, in which each group is a bin and each bin's free space is the difference between the group's height and the target makespan.

The algorithm begins by assigning all tasks the maximum frequency $F_{\max }$ and computing the initial height height $\left(F_{\max }\right)$ of all groups $i$. All tasks $j$ are queued after their decreasing running time $t t_{j}$ at frequency $f_{j}$ (initially $\forall j: f_{j}=F_{\max }$ ). We call $f_{j, \text { cur }}$ any frequency assigned to task $j$ at any point in the algorithm. We call $F_{\text {next }}$ the frequency variable that iterates over the set of frequencies $F$ sorted decreasingly. For all tasks $j$ in the task priority queue, if $f_{\text {next }}<f_{j, \text { cur }}$ and the time penalty resulting in running task $j$ at frequency $f_{\text {next }}<f_{j \text {,cur }}$ is lower than or equals the gap between the height height $i_{j}$ of the group $i_{j}$ (to which task $j$ is mapped) and the target makespan $M$ (i.e., if $t t_{j}\left(w_{j}, f_{\text {next }}\right)-t t_{j}\left(w_{j}, f_{j, \text { cur }}\right) \leq M-$ height $\left._{i_{j}}\right)$, then we can assign task $j$ the frequency $f_{\text {next }}$, update the height of group $i_{j}$ as described in Section 3.3, and insert the newly scaled task into a new task priority queue for the next frequency iteration.

The initial frequency assignment to tasks takes $O(n)$ steps, and the computation of the initial height for each group takes $O(n)$ for each $O(p)$ group, hence $O(p n)$. Tasks and frequencies are sorted decreasingly in $O(n \log n)$ and $O(|F| \log |F|)$ time steps, respectively. In each nested loops iteration, taking the longest running time from the sorted task queue takes $O(1)$ time steps. Checking if running task $j$ at frequency $f_{\text {next }}$ does not make the schedule to cross the target makespan $M$ takes $O(1)$ time steps if the height of group $i_{j}$ is already computed. Updating the height of group $i_{j}$ takes $O(p)$ time steps and adding the task in the new priority queue takes $O(\log n)$ time. Since we have $|F|$ frequency levels and $n$ tasks, we run $n \cdot|F|$ iterations. Therefore, the Height heuristic runs in $O(n+p n+n \log n+|F| \log |F|+p n \cdot|F| \log n)=O(|F| \cdot p n \log n)$ time steps.

\subsection{Binary Search Allocation Heuristic}

These methods described for the allocation, mapping and frequency scaling phases, rely on the mapping phase capability to compute task distribution close to optimal and produce a schedule whose runtime is the average load that each core receives (Eq. (5)). 
$\mathrm{M}$

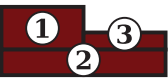

(a) Initial schedule: all tasks run at $f=$ $\max (\{1,2,3\})=3$.
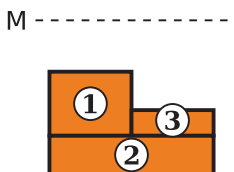

(b) Scale tasks 1, 2 and 3 at $f_{\text {next }}=2$. queue before states of Figure 5(a) and after Figure 5(b).

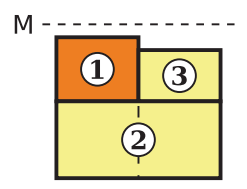

(d) Only tasks 2 (e) Final task priorand 3 can scale fur- ity queue . ther at $f_{\text {next }}=1$.

Fig. 5. Running example of 3 tasks scaled with the Height heuristic for 2 processors of 3 frequency levels.

However, we target the minimization of energy consumption under makespan constraint instead of runtime. As parallelization usually induces additional processing cost due to a task $j$ 's nonperfect efficiency function $e_{j}(q) \leq 1$, a too-high parallelization consumes more energy. This suggests giving tasks as little parallelism as possible [Belkhale and Banerjee 1990].

We set a minimal acceptable efficiency value $e_{\min }$ for allocations of processors to all tasks, in order to limit parallelization and reduce its cost. We run the fast allocation, with the additional constraint that no task $j$ should be allocated a number of cores that yields $e_{j}<e_{\min }$. The constraint is implemented as an $O(1)$ condition to discard any allotment $q$ for a task $j$ if $e_{j}(q)<e_{\min }$ in the search for $q$ that minimizes $t t_{j}(q, 1)$. Then, we use the fast heuristics described in Sections 3.3 and 3.5 to check if the limited parallelization still allows our heuristics to compute a valid schedule. If so, we increase the minimal efficiency threshold by $\Delta$ and compute a new schedule. Otherwise, we decrease the minimal efficiency by $\Delta$. Finally, we update $\Delta$ to its half. We implement a binary search on $e_{\min }$ to maximize the minimal efficiency in the search space $(0,1)$, with an initial threshold 0.5 and $\Delta=0.25$ (Figure 3(b)). The binary search stops when the minimal efficiency is higher than any possible value for $e_{j, w_{j}}\left(W_{j}>1\right)$ for all tasks $j$ (Figure 3(b), case 1) or when $\Delta$ becomes lower than the minimal efficiency difference between two allocations for any task.

The binary search sequentializes tasks as far as task mapping and frequency scaling allow the production of valid schedules. However, more sequential tasks take longer time to execute. This can result in the frequency scaling phase to increase the task's frequency, which can replace the parallelization overhead by a much higher frequency cost, due to the fast increase of $P C(f)=f^{\alpha}$. During the binary search, we keep track of the energy consumption of every schedule generated and we keep the best. The best initial solution is computed with fast allocation (Section 3.1) with no efficiency constraint $\left(e_{\min }=0\right)$. Before starting our binary search, we also try an allocation with a perfect efficiency constraint $e_{\min }=1$, that is only possible with sequential or perfectly scalable tasks. If such an allotment results in a schedule running all tasks at the lowest possible frequency, then we select this solution and skip the search loop. Otherwise, we compare it to the initial best solution and begin the binary search iterations.

Figure 3(b) illustrates four possible search scenarios. In scenario 1, a progressive increment makes the final schedule's makespan approach $M$, but it never reaches or crosses it. All allocations are valid but the best one can be any of them (bottom curve, plain black squares). In scenario 2 , the initial $e_{\min }$ doesn't allow subsequent heuristics to compute a valid schedule. $e_{\min }$ is drastically reduced by initial $\Delta$, and the resulting schedule's makespan is much lower than the target. The binary search method continues to halve $\Delta$, increasing and decreasing $e_{\min }$ by $\Delta$ until $\Delta$ is too small and the allocation that yields the best schedule is kept (mid-dark green rounds). In scenario 3 , the binary search increments $e_{\min }$ so that the final schedule approaches $M$ as in scenario 1 ; but as $e_{\min }$ increases, the heuristics begin to fail at computing 
a valid schedule. Binary search behaves then as in scenario 2 , until $\Delta$ is too small (dark-red diamonds). Finally, in scenario 4, the binary search may always decrease $e_{\min }$ but still fails to produce any allocation that the heuristics could use to compute any valid schedule (top light-blue triangles). In this case, we use the initial best solution computed with the fast allocation strategy (Section 3.1) and rely on LTLG and Height heuristics to compute a valid schedule, if possible.

The binary search algorithm explores the search space $(0,1)$ for $\Delta$. Each round runs the LTLG $(O(n p \log p))$ and Height $(O(|F| \cdot p n \log n))$ heuristics and run therefore in $O(n p(\log p+|F| \cdot \log n))$ time steps. We define the stop condition as $\Delta$ being smaller than the efficiency gap between two allocations for all tasks, which we calculate before beginning the binary search. The minimal allocation gap takes time $O(n \log p)$ to compute (see Section 3.1 on allocation), where allocations that exhibit too low efficiency are pruned during search. If $g$ is the minimal efficiency gap between two possible allocations for any task (a large value of $g$ means that all tasks scale badly), then $\gamma=\left\lceil\log _{2}(\Delta / g)\right\rceil$ is the number of rounds in our binary search. We also compute twice a fast allocation (time $O(n \log p)$ ) as an initial solution and attempt with a perfect efficiency constraint. The overall complexity of our binary search is therefore $O\left(n \log p+2(n \log p+n p \log p+|F| \cdot p n \log n)+\left\lceil\log _{2}(\Delta / g)\right\rceil \cdot n p(\log p+|F| \cdot \log n)\right)=$ $O\left(\left\lceil\log _{2}(\Delta / g)\right\rceil \cdot n p(\log p+|F| \cdot \log n)\right)$.

\subsection{Simulated Annealing}

The binary search method described in Section 3.6 limits the allocation space exploration to solutions in which all tasks share a common efficiency constraint. In particular, it does not permit any fine tuning through individual tasks' allotment adjustment. We use the fast LTLG mapping and height frequency scaling heuristics within a simulated-annealing meta-heuristic that optimizes the core allocation for each task. Our simulated annealing thus optimizes allocation for a task collection.

We define the neighborhood of an allocation as other allocations within a fixed distance. The distance between two allocations is a real in the interval $[0,1]$, that defines a proportional amount of the $n$ tasks, whose number of cores allotted differs between both allocations. The distance also defines the proportional variation of number of cores, as a power of 2, that run the same task in both solutions. Searching in the neighborhood of an allocation within a distance 0 , is searching in the set of all allocations whose exactly one task is allocated the next higher or lower power-of-2 number of cores, compared to the allocation of origin. Searching in the neighborhood of an allocation within a distance 1 is searching in all possible allocations. A search within the neighborhood of an allocation always excludes the allocation of origin.

We begin with a schedule computed with binary search (Section 3.6). The LTLG mapping and height frequency scaling heuristics compute a schedule for this allocation; we use the resulting energy consumption (Equation (4), $P C(f)=f^{\alpha}, \alpha=3$ ) as feedback to the simulated annealing allocation phase. In our experiment, we search a new solution within the neighborhood of the current allocation at a maximal distance 0.5 at each simulated annealing iteration. We allow at most 2 iterations before we decrease the simulated annealing temperature (starting at 8) by multiplying it by our cooling factor 0.6. For each temperature level, we accept at most 2 improving solutions. The simulated annealing stops when the simulated temperature reaches 0.9 , or whenever it finds a correct solution where all tasks are sequential and run at the minimal frequency.

We have $\lceil\log (8 / 0.9) / \log (1 / 0.6)\rceil=5$ iterations until the final temperature is reached. We allow at most 2 transformations per iteration, resulting in at most $10=O(1)$ runs of the task mapping and frequency scaling phases. Because we use binary search as an initial solution, the overall time complexity is $O\left(n p\left(\log p+|F| \cdot \log n+\left\lceil\log _{2}(\Delta / g)\right\rceil\right)+\right.$ $10(p n(\log n+\log p))=O\left(n p\left(\log p+|F| \cdot \log n+\left\lceil\log _{2}(\Delta / g)\right\rceil\right)\right)$. 


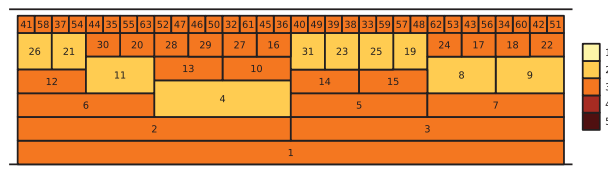

(a) Optimal phase-separated crown schedule.

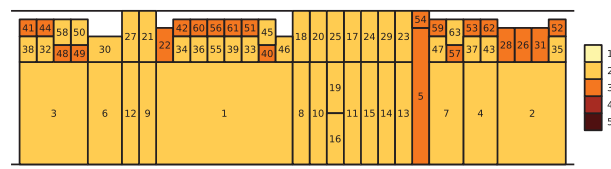

(b) Optimal integrated crown schedule.

Fig. 6. Phase-separated and integrated solutions; the lighter the tasks, the lower their running frequency. Optimal allocation used in the phase-separated approach restricts the quality of the final solution. Frequencies are represented by colors and darkness: dark red is high frequency (5) and lighter yellow represents low frequency (1).

\section{INTEGRATED ENERGY-EFFICIENT CROWN SCHEDULING}

Separate crown allocation, crown mapping, and a-posteriori scaling of the resulting crown schedule may lead to suboptimal results compared to co-optimizing resource allocation, mapping, and discrete scaling from the beginning. See Figure 6 for an example.

The integration of crown allocation, mapping, and scaling is modeled as follows: We construct an integer linear program that uses $(2 p-1) \cdot s \cdot n$ binary variables $x_{i, k, j}$ where $x_{i, k, j}=1$ denotes that task $j$ has been assigned to group $i$ and should run at frequency level $F_{k}$. We require that each task be allocated and mapped to exactly one group and frequency level:

$$
\forall j=1, \ldots, n: \sum_{i=\max \left(p / W_{j}, 1\right)}^{2 p-1} \sum_{k=1}^{s} x_{i, k, j}=1 .
$$

and forbid the mapping of a task with width limit $W_{j}$ to an oversized group:

$$
\forall j=1, \ldots, n: \sum_{i=1}^{\max \left(p / W_{j}, 1\right)-1} \sum_{k=1}^{s} x_{i, k, j}=0 .
$$

Another constraint asserts that no processor is overloaded:

$$
\forall m^{\prime}=1, \ldots, p: \text { time }_{m}:=\sum_{i \in C_{m}} \sum_{k=1}^{s} \sum_{j=1}^{n} x_{i, k, j} \cdot \frac{\tau_{j}}{p_{i} e_{j}\left(p_{i}\right) F_{k}} \leq M .
$$

Then we minimize the target energy function (as derived from Eq. (4)):

$$
\sum_{i=1}^{2 p-1} \sum_{k=1}^{s} P C\left(F_{k}\right) \cdot \sum_{j=1}^{n} x_{i, k, j} \cdot \frac{\tau_{j}}{e_{j}\left(p_{i}\right)}
$$

Note that the coefficients $\tau_{j}, p_{i}$ and $e_{j}\left(p_{i}\right)$ are constants in the linear program. Note also that the time and energy penalty for frequency switching is not modeled.

One sees that $(2 p-1) \cdot s \cdot n$ variables and $p+n$ constraints are needed to allocate, map, and scale $n$ tasks onto $p$ cores with $s$ frequency levels.

\section{DYNAMIC CROWN RESCALING}

The methods for crown resource allocation, mapping, and scaling optimize for the steady state of the pipeline, in particular, for the "worst" case that all tasks are data ready and execute in each round. In practice, however, there might be cases in which individual tasks are not yet data ready at the issue time and thus are to be skipped for the current round by the crown scheduler, or in which tasks are delayed due to cache misses, branch mispredictions, or unexpected I/O latency. 
If the skipped or delayed task $j$ was mapped to a group (even a singleton group) $G_{i}$, for instance, the black hashed task in Figure 3(a), we call $\beta$ the additional execution time of group $i$ due to variable delays or tasks skipped. If task $j$ is skipped, then the additional execution time is the negative opposite of $t t_{j}\left(\beta=-t t_{j}<0\right)$.

If $\beta<0$, we can save more energy, or if $\beta>0$, the schedule may not meet the target makespan $M$. We can rescale "down-crown" all $n^{\prime} \leq n$ remaining tasks of the processors in $G_{i}$ that follow $j$ in the crown schedule (gray hashed area in Figure 3(a)). Note that such rescaling must be consistent across all processors sharing a moldable task, thus the concurrent search for candidates to rescale must be deterministic. The crown structure can be leveraged for this purpose. Note that if a task of the root group is skipped or delayed, then the entire schedule needs to be rescaled.

Dynamic rescaling of the current round is an online optimization problem that should be solved quickly such that its expected overhead remains small in relation to the saved amount of time. Thus, simple heuristics should be appropriate here. Let $R_{i}$ be the set of tasks in group $i$ already executed $\left(j \notin R_{i}\right)$. We can apply the Height heuristic (Section 3.5) with the subgroup $G_{i}$ running tasks in $R_{i}$ as the root group and target the makespan

$$
M^{\prime}=M-t t_{j}-\beta-\sum_{x=1}^{\left\lfloor\log _{2} i\right\rfloor} g t_{\left\lfloor i / 2^{x}\right\rfloor}-\sum_{j^{\prime} \in R_{i}} t t_{j^{\prime}}
$$

for $p^{\prime}=\left|G_{i}\right|$ processors $\left(p^{\prime} \leq p\right)$ and $n^{\prime}<n$ tasks, taking $O\left(|F| p^{\prime} n^{\prime} \log n^{\prime}\right)$ time steps.

\section{EXPERIMENTAL EVALUATION}

We implemented the ILP models in AMPL [Fourer et al. 1993]; we implemented the LTLG mapping, height frequency scaling, binary search, and simulated annealing allocation heuristics in C++. We substitute the ILP-based mapping and frequency scaling phases of the phase-separated variant [Kessler et al. 2013b] with an optimal loadbalanced ILP-formulated mapping phase, the LTLG mapping, and with the Height mapping heuristics, respectively. We obtain and compare 7 different crown schedulers:

(1) ILP allocation, ILP load-balanced mapping, ILP frequency scaling (Fast,Bal.ILP, ILP)

(2) ILP allocation, LTLG mapping heuristic, ILP frequency scaling (Fast,LTLG,ILP)

(3) ILP allocation, ILP load-balanced mapping, Height heuristic frequency scaling (Fast,Bal.ILP,Height)

(4) ILP allocation, LTLG mapping heuristic, Height frequency scaling heuristic (Fast,LTLG,Height)

(5) Binary search with LTLG mapping and Height frequency scaling heuristics (Bin,LTLG,Height)

(6) Simulated annealing allocation with initial binary search, LTLG mapping and Height frequency scaling heuristics (Bin,LTLG,Height Ann.)

(7) ILP integrated (Integ.)

We also implemented an ILP formulation from Xu et al. [2012], whose scheduler arranges tasks in levels of strips spanning across cores. Each level's height is defined by a key task, that is, the first task mapped to its level. The rectangle of cores unused by the key task of a level is 2D-packed using some of the tasks not scheduled yet. Frequency scaling is performed through the rescaling of entire levels, that is, the rescaling of all tasks mapped to this level, as a bin packing problem. Finally, we adapted and implemented the NLP (Non-Linear Programming) formulation from Pruhs et al. [2008] in AMPL and their heuristic in C++. Their technique schedules sequential tasks only (and therefore it never parallelizes any task $j$ regardless of its maximal parallel degree $W_{j}$ ) 
and scales tasks' frequency with a continuous and unbounded frequency domain. For the NLP implementation, we perform frequency scaling using our ILP-based method (Section 3.4). For the heuristic, we use best-fit to assign discrete and bounded frequency levels to tasks.

For small and medium problems and machine sizes, we generate synthetic task collections as follows: we group synthetic task collections in different categories defined by the number of cores $(p \in\{1,2,4,8,16,32\})$, the number of tasks $(10,20,40$ and 80 tasks), and tasks' maximum widths $W_{t}$ : sequential $\left(W_{t}=1\right.$ for all $\left.t\right)$, low $\left(1 \leq W_{t} \leq p / 2\right)$, average $\left(p / 4 \leq W_{t} \leq 3 p / 4\right)$, high $\left(p / 2 \leq W_{t} \leq p\right)$ and random $\left(1 \leq W_{t} \leq p\right)$. Tasks' maximum width are distributed uniformly. The target makespan of each synthetic task collection is the mean value between the runtime of an ideally load-balanced task collection running at lowest frequency and at highest frequency:

$M_{s y n}=\sum_{j=1}^{n} \frac{3 \cdot t t_{j}(1,1)}{8 \min \left(W_{j}, p\right) F_{\min }}+\frac{t t_{j}\left(\min \left(W_{j}, p\right), 1\right)}{8 \cdot F_{\min }}+\frac{3 \cdot t t_{j}(1,1)}{8 \min \left(W_{j}, p\right) F_{\max }}+\frac{t t_{j}\left(\min \left(W_{j}, p\right), 1\right)}{8 \cdot F_{\max }}$

We use the same scheme to generate large problems and machine sizes, ranging from 500 to 2000 tasks and from 256 to 1024 processors for tasks of a random maximum width.

We also provide task collections of classic streaming algorithms: parallel FFT, parallel-reduction and parallel mergesort. FFT is characterized by $2 \cdot p-1$ parallel tasks of a balanced binary tree. In level $l \in\left[0 ; \log _{2} p\right]$ of the tree, there are $2^{l}$ dataparallel tasks of width $p / 2^{l}$ and work $p / 2^{l}$ so that all tasks could run in constant time. The mergesort task collection is similar to FFT, but all its tasks are sequential. Parallel reduction involves $\log _{2} p+1$ tasks of maximum width $2^{l}$ and work $2^{l}$ for $l \in\left[1 ; \log _{2} p+1\right]$; they can also run in constant time. Because these task collections yield perfectly balanced task work and maximum widths, they are easy to schedule with a loose target makespan as defined for the synthetic task collections. We use a more constraining target makespan for concrete task collections. The makespan for FFT is

$$
M_{F F T}=\sum_{j=2}^{n} \frac{t t_{j}\left(1, F_{\min }\right)}{2 \cdot p}+\sum_{j=2}^{n} \frac{t t_{j}\left(1, F_{\max }\right)}{2 \cdot p}
$$

that is, the makespan of a synthetic task collection, ignoring task 1 . The makespan for parallel mergesort,

$$
M_{m s o r t}=\frac{\max _{\forall j}\left(\tau_{j}\right)}{\max F},
$$

takes the root merging task and divides its work by the maximal frequency available. The makespan for parallel reduction task collections,

$$
M_{\text {preduce }}=\sum_{j=1}^{n} \frac{\tau_{j}}{2 \cdot \min \left(p, W_{j}\right) \cdot F_{\min }}+\sum_{j=1}^{n} \frac{\tau_{j}}{2 \cdot \min \left(p, W_{j}\right) \cdot F_{\max }}
$$

is the same as for synthetic collections, but we assume perfect scalability. All our synthetic and classic task collections have a minimal efficiency gap of $g=0.023438$, yielding a complexity factor of $\gamma=\left\lceil\log _{2}(0.5 / 0.023438)\right\rceil=5$ for our binary search method.

Finally, we use the technique of Gordon et al. [2006] to extract tasks' workload and parallel efficiency from the Streamit benchmark suite. We schedule all variants of the applications audiobeam, beamformer, channelvocoder, fir, nokia, vocoder, BubbleSort, 
filterbank, perftest and tconvolve from the Streamit compile source package ${ }^{8}$. We use their compiler to obtain for each task $j$ the task's estimated workload $\tau_{j}$, the task's parallel degree $W_{j}$ (either 1 or $+\infty$ ) and the task's communication rate we call $\psi_{j}$. We use $\psi_{j}$ to compute tasks $j$ 's parallel efficiency $e_{j}(q)$ (Eq. 14):

$$
e_{j}(q)= \begin{cases}1 & \text { if } q=1 \\ \tau_{j} /\left(\tau_{j}+q \cdot \psi_{j}\right) & \text { if } q>1 \text { and } q \leq W_{j} \\ 10^{-6} & \text { otherwise }\end{cases}
$$

and we use Equation (10) to compute a target makespan.

We run both the ILP solver and heuristics on a quad-core i7 Sandy Bridge processor at $3 \mathrm{GHz}$ and $8 \mathrm{~GB}$ of main memory. We use Gurobi 5.10 and ILOG AMPL 10.100 with 5 minutes timeout to solve the ILP models, and compile our $\mathrm{C}++$ implementations with gcc 4.6.3 and -O3 optimization switch, on Ubuntu 12.04. Gurobi exploits 8 hardware threads and our heuristics are implemented as sequential programs.

We measure the overall scheduling quality (energy consumption) of all 7 crown schedulers as well as schedulers from Xu et al. [2012] and Pruhs et al. [2008]. Figures 7(a) and 7 (b) show mean values for 10, 20, 40, and 80 tasks and across all width classes $\{$ Random, Serial, Low, Average, High\} for the synthetic task collection. Figures 8(b) and $9(\mathrm{~b})$ show the average energy consumption by task collection classes for the classic streaming algorithms and the Streamit benchmark, respectively. We can see that the integrated crown scheduler-as well as scheduler heuristics based on binary search, LTLG, Height and simulated annealing combined - consistently produce the best schedules. NLP and heuristics from Pruhs et al. [2008] perform well on larger architectures and with sequential task sets (Figures $7(\mathrm{~b})$ and 8(b)), but its solutions' quality drop when tasks' parallel degrees increase (Figures 7(a), and 8(b)). The level-packing solution from Xu et al. [2012] performs mostly poorly, except for a small amount of tasks, or for sequential tasks. An exception is parallel reduction (Figure 8(b)), for which it performs at least as good as our integrated, ILP-based crown scheduler. We scheduled a subset of our synthetic task collection set with random workload variations within $1 \%$ for each task and found no significant energy variations in schedules. Finally, we show for the classic streaming task collection and the Streamit benchmark suite the schedulers' ability to compute a valid schedule in Figures 8(a) and 9(a). These figures show that Pruhs's techniques fail at scheduling parallel tasks under the makespan constraints we impose, while Xu's succeeds as much as our crown schedulers. For sequential task collections such as for mergesort, our crown scheduler has the same success rate as Pruhs's, whereas Xu's approach fails more often.

We also compare the makespan produced by our LTLG mapping heuristic to our crown-optimal, load-balanced ILP mapping formulations (variant 1 presented earlier) as well as our binary search and simulated annealing heuristics and our implementations of Xu's and Pruhs's techniques (Figure 7(c)). Similarly, we compare in Figure 7(d) the efficiency of the frequency scaling ILP formulation and Height heuristic to optimize energy through frequency scaling. In order to ensure a fair comparison between both ILP and Height heuristic frequency scaling implementations, we provide both of them with a mapping obtained by different runs of our crown-optimal allocation and mapping ILP formulations, as a problem input for frequency scaling. Figure 7(c) shows that our LTLG heuristic is very competitive with our crown-optimal ILP load-balancing formulation. The same figure suggests that the initial allocation influences greatly the mapping makespan. Our ILP and LTLG heuristic use the fast allocation (Section 3.1), that is,

\footnotetext{
${ }^{8}$ At the time of writing, the latest version was pushed to github on Aug 3, 2013. See groups.csail.mit.edu/ $\mathrm{cag} /$ streamit/restricted/files.shtml and the source package for information about these applications.
} 


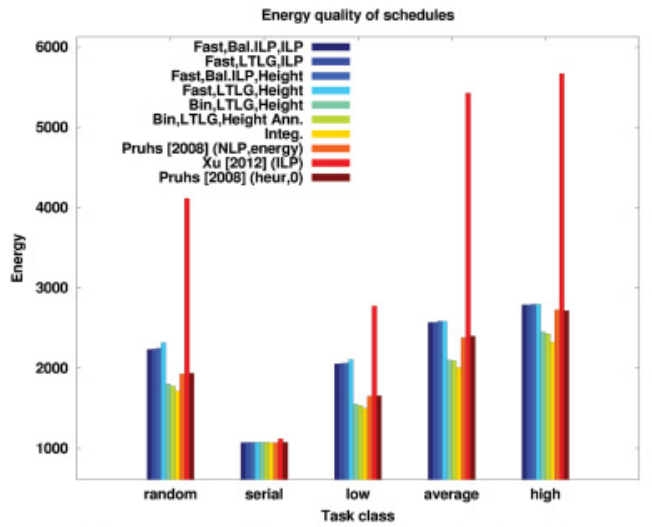

(a) Energy prediction by task collection class.

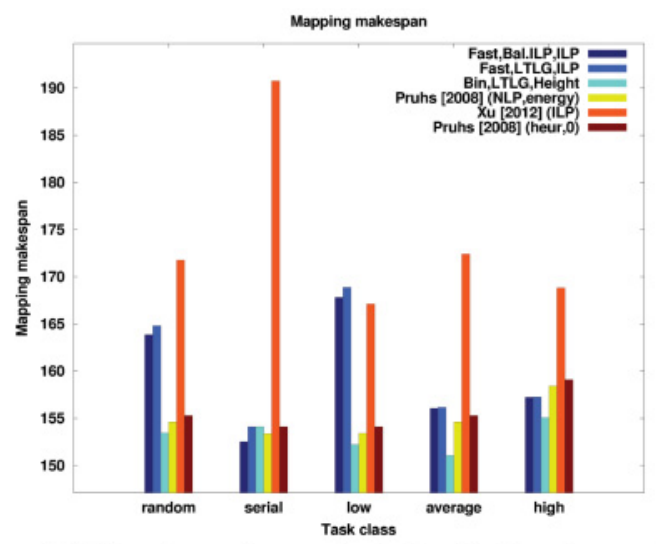

(c) Mapping makespan by task collection class.

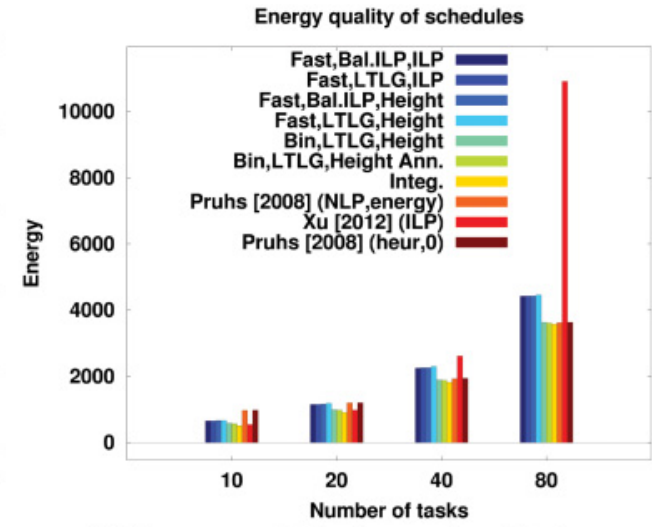

(b) Energy prediction by number of tasks.

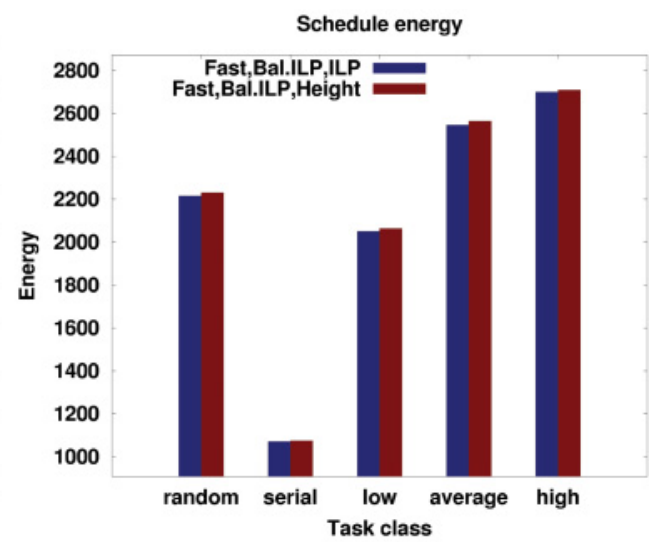

(d) Frequency scaling energy by task collection class.

Fig. 7. Projected energy per number of cores and tasks and efficiency of LTLG and Height heuristics for the synthetic task collection.

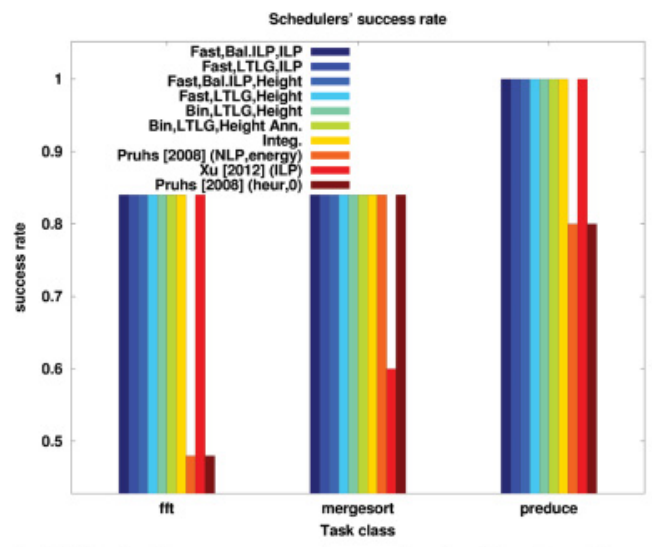

(a) Scheduling success rate per task collection class.

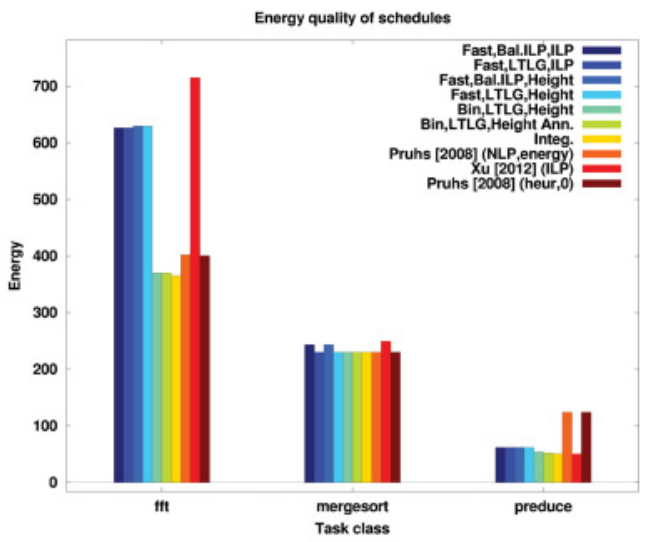

(b) Energy prediction by task collection class.

Fig. 8. Scheduling success rate and projected energy for FFT, mergesort, and parallel reduction. 


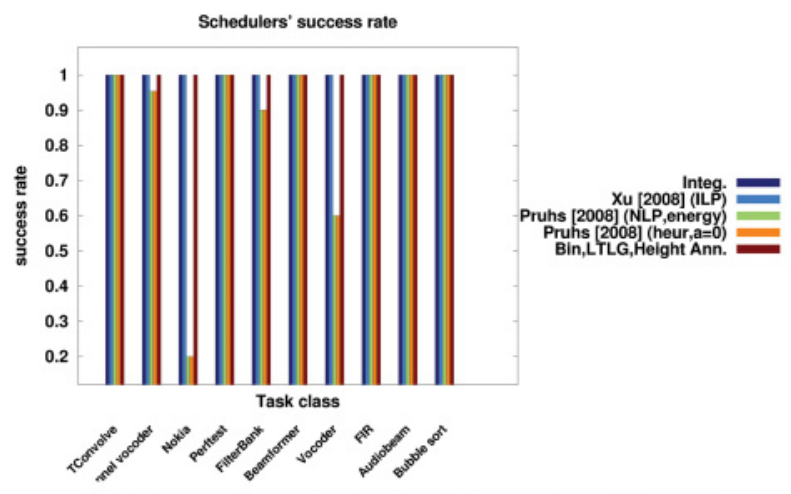

(a) Scheduling success rate per task collection class.

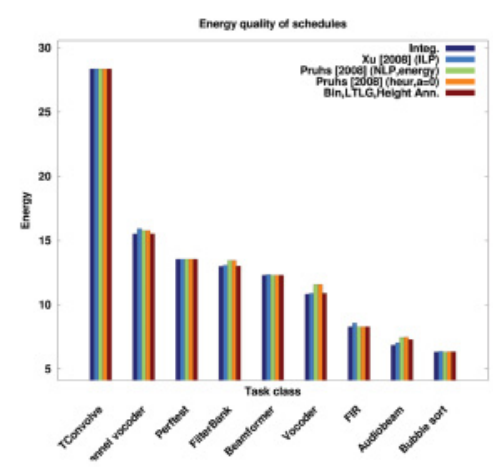

(b) Energy prediction by task collection class (logarithmic scale).

Fig. 9. Scheduling success rate and projected energy for FFT, mergesort, and parallel reduction.

allocating as many processors as possible to tasks. In contrast, Pruhs's NLP formulation and heuristic schedule sequential tasks. As a consequence, Pruhs's makespans are much lower for tasks that cannot run on many processors (but can run on a few), while the situation is more balanced with highly parallel tasks. The binary search always finds another allocation that allows lower makespan than either our crown heuristic with fast allocation or Pruhs's solutions. Again, the approach from Xu et al. [2012] yields much higher makespans. Figure 7(d) demonstrates the efficiency of our Height heuristic compared to our crown-optimal, ILP-based frequency scaling method.

We provide the measured overall optimization time for all scheduler variants for the synthetic task collection set by number of tasks and number of cores (Figures 10(c) and 10(f)), as well as the optimization time for both mapping and frequency scaling phases (Figures 10(a), 10(d), 10(b) and 10(e)). We compare the measured optimization time of our heuristics and their time complexity by number of tasks and number of cores in Figures 11 and 12 for small, medium, and large problems of the synthetic task set. We can see that Pruhs's, Xu's, and our crown integrated ILP and NLP formulations largely dominate the overall optimization time. Phase-separated, crown-optimal ILP crown schedulers yield a much lower optimization time and none of the heuristics is visible in Figures 10(c) and 10(f). The six figures in Figure 10 demonstrate that the LTLG and Height heuristics run much faster than their crown-optimal ILP counterparts, and that the phase-separated crown schedulers' optimization time is dominated by the mapping phase. Figures 11(a), 11(b), 12(a), and 12(b) demonstrate that the optimization time for heuristics is dominated by the simulated annealing and our binary search crown schedulers. The phase-separated LTLG and Height crown heuristic as well as Pruhs's heuristic perform much faster. This can be explained by their lack of an elaborate allocation phase that our binary search and simulated annealing implement as a feedback loop. However, such a simplistic allocation strategy makes them produce a worse solution, or to fail at producing any valid one. Finally, Figures 11(a), 11(b), 11(c), and 11(d) show that our heuristics' optimization times follow the optimization time predicted by our complexity analysis. However, this is not true for our simulated annealing and binary search with the large synthetic task collection and target architectures (Figures 12(a), 12(b), 12(c), and 12(d)). This can be due to the search interruption when a valid schedule is found where all tasks run sequentially at the lowest frequency available.

The crown constraints exhibit no restrictions to map sequential tasks to processors, but they allow the mapping of some parallel tasks. Hence, the solution space of Pruhs's mapping technique is strictly included in our crown mapping's solution space. Also, 

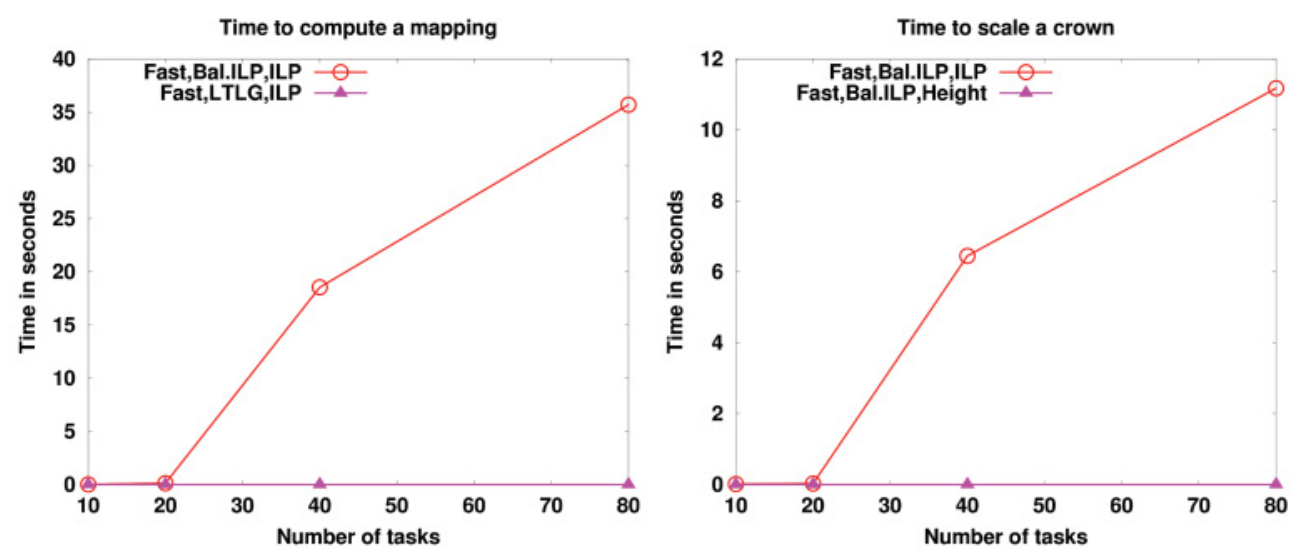

(a) Mapping phase runtime by number of tasks.

(b) Frequency scaling phase runtime by number of tasks.

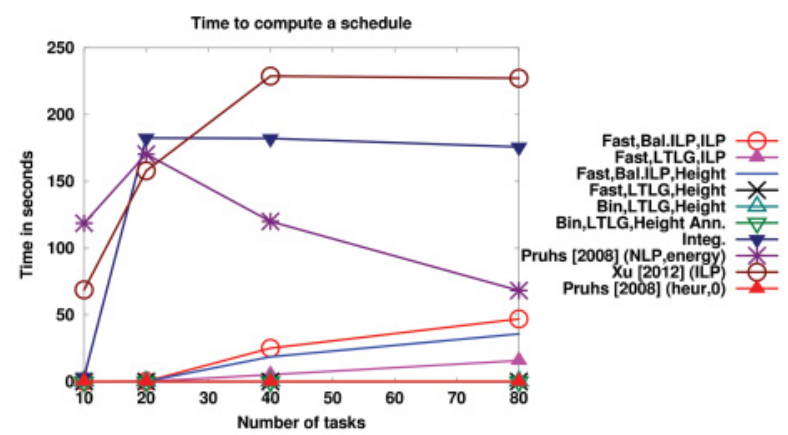

(c) Overall optimization time by number of tasks.

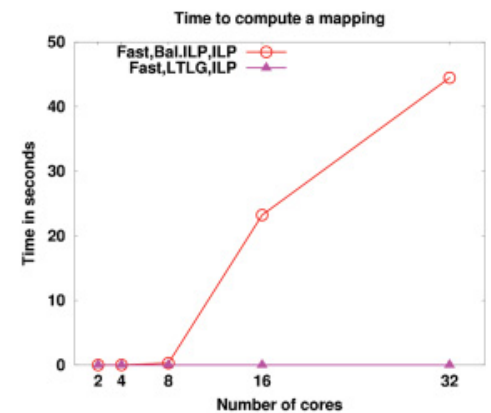

(d) Mapping phase runtime by number of cores.

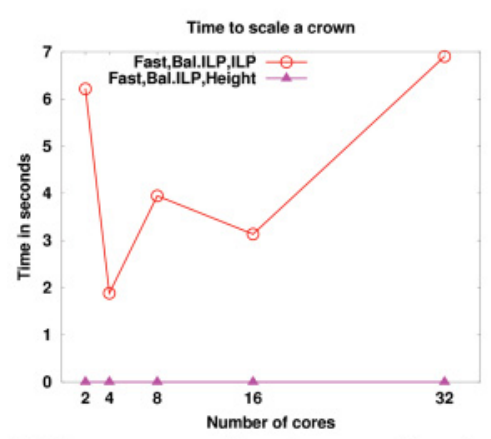

(e) Frequency scaling phase runtime by number of cores.

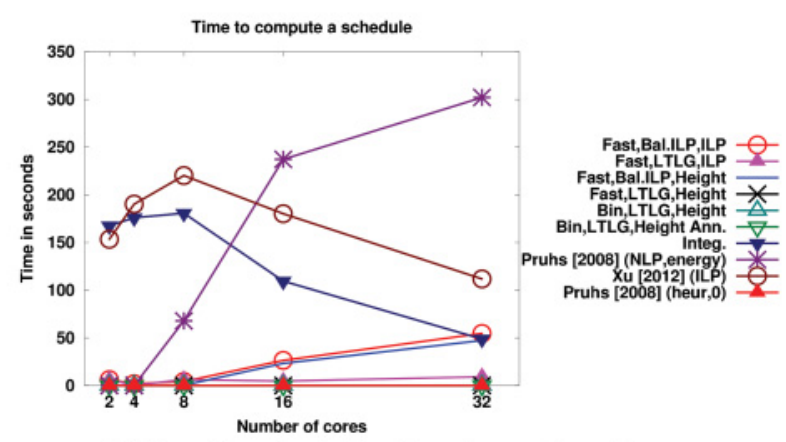

(f) Overall optimization time by number of cores.

Fig. 10. Share of mapping and frequency scaling phases over overall optimization time.

since we implemented the same ILP frequency scaling method for both schedulers, the overall solution space of crown schedulers includes the one of our implementation of Pruhs's. Therefore, when both formulations can be solved to optimality, the integrated crown scheduler will always find a solution at least as good as Pruhs's. Similarly, our LTLG heuristic for sequential tasks is reducible to LPT. Since we use LPT in our implementation of Pruhs's heuristic, its solutions cannot be better than LTLG's. Again, the Height heuristic is an implementation of best-fit for the crown structure; therefore 


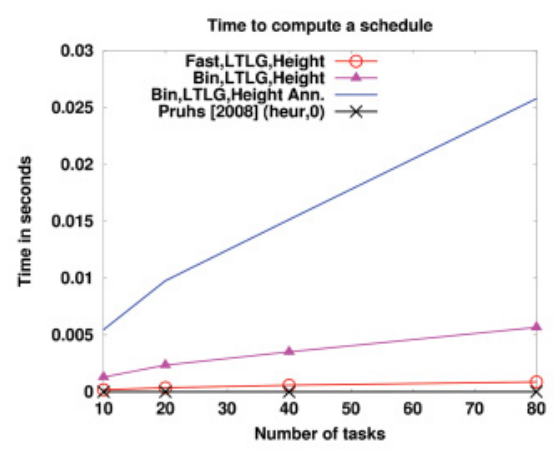

(a) Overall optimization time per number of tasks.

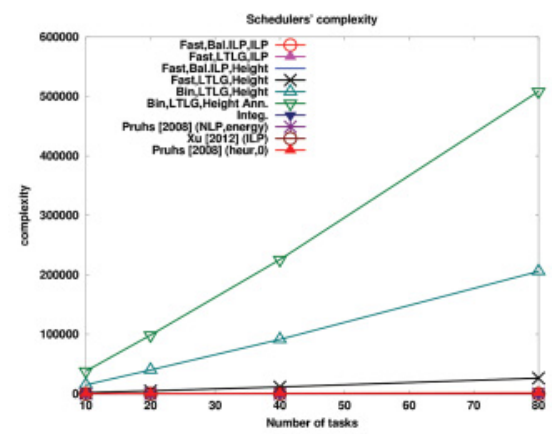

(c) Overall complexity per number of tasks.

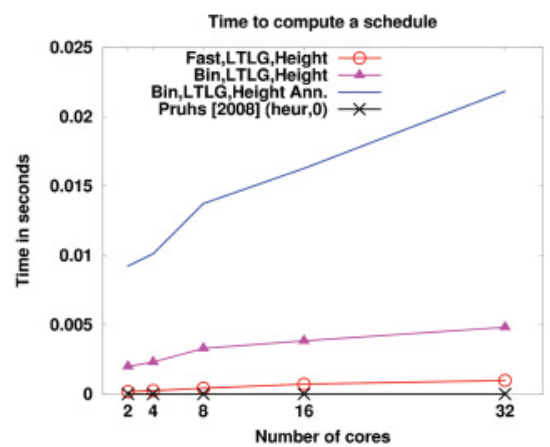

(b) Overall optimization time per number of cores.

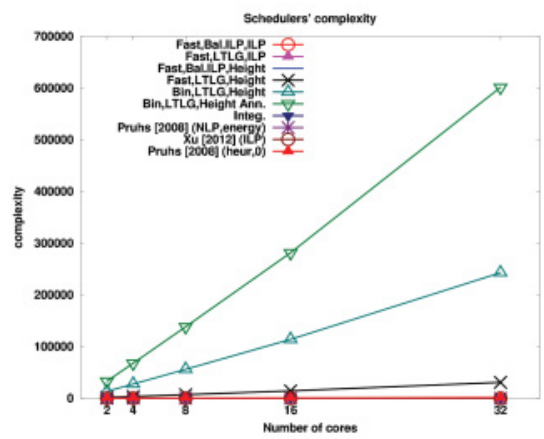

(d) Overall complexity per number of cores.

Fig. 11. Measured optimization time and time complexity of schedulers.

it has the same solution space as Pruhs's frequency scaling method. Since our LTLG and Height heuristics are reducible to Pruhs's mapping and frequency scaling methods, they can always find a solution at least as good.

We inspected schedules of the synthetic task sets produced by our best crown scheduler heuristic as well as the technique described by Pruhs et al. [2008]. We assume that tasks in our synthetic task set run sequentially and at the lower frequency available for between $1 \mathrm{~ms}$ and $19 \mathrm{~ms}$, that is, $8 \mu \mathrm{s}$ with 32 cores at frequency 5 . We assume further that switching frequency takes $50 \mu \mathrm{s}$ [Mazouz et al. 2014] and $5 \cdot 10^{-6}$ energy units. Finally, we assume that frequency switching can take place asynchronously, while a task is running [Mazouz et al. 2014]. We found that all frequency transitions can be hidden when tasks are processed or when processors are idle. In the case of a task running for too short a time to hide a necessary frequency transition, no significant additional time and energy was found.

\section{RELATED WORK}

Energy-aware allocation, mapping, and scheduling problems are being researched intensively in scheduling theory. For instance, Edmonds and Pruhs [2012] consider an online scheduling algorithm for jobs arriving over time that minimize overall response time (the sum of distances between arrival and completion of each job). Jobs are parallelizable with arbitrary speedup curves, but frequency scaling or energy does not play a role. Chan et al. [2011] use an analytic power model and continuous frequencies. Their algorithm does not statically know the work of a job, nor its speedup curve. 


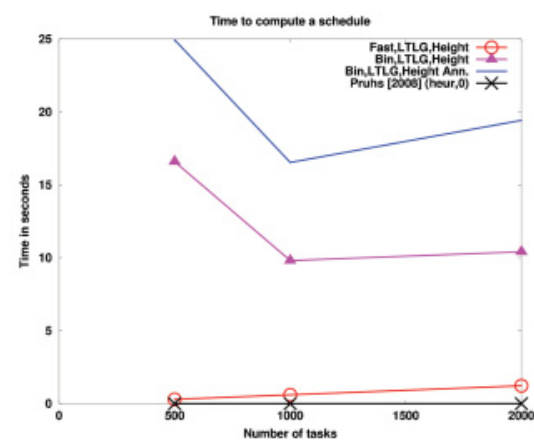

(a) Overall optimization time by number of tasks.

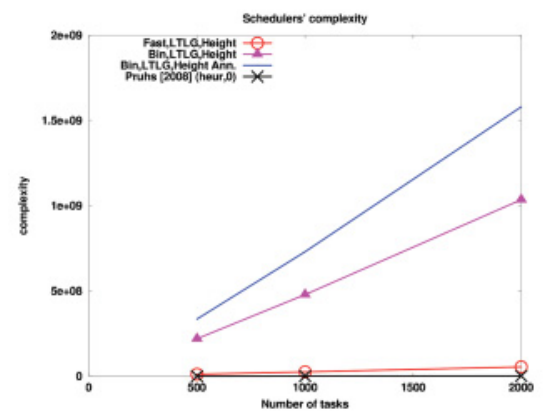

(c) Overall complexity by number of tasks.

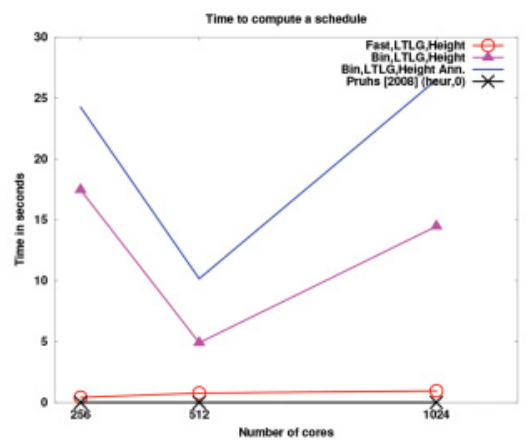

(b) Overall optimization time by number of cores.

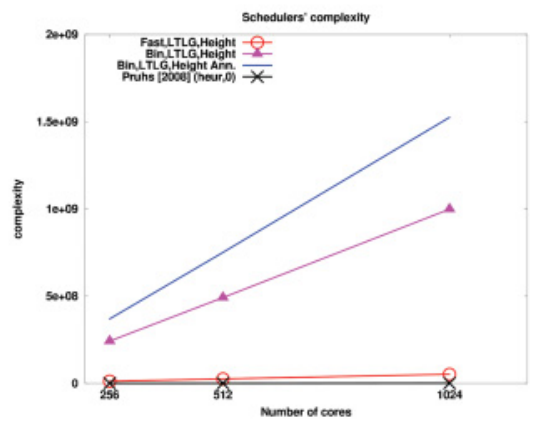

(d) Overall complexity by number of cores.

Fig. 12. Overall scheduling time and energy consumption of the resulting crown schedules for large task collections and massively parallel architectures.

Energy-efficient static scheduling of task collections with parallelizable tasks onto multiprocessors with frequency scaling has been considered by Li [2012]. Li's power model is defined as polynomially reducible to $f^{\alpha}$ where $\alpha \geq 3$ and therefore comparable to the one we use in this article. However, the number of processors allocated to each task is fixed and given as part of the problem scenario, and continuous (not discrete) frequency scaling is used.

$\mathrm{Xu}$ et al. [2012] use a level-packing approach, which means that they might need as many conceptual barrier synchronizations as they have levels, that is, up to the number of jobs. They also use discrete frequency levels, arbitrary power profiles of cores, moldable tasks with individual, arbitrary speedup curves, and ILP. As their ILP approach needs many more variables than ours, they do not use a solver but only evaluate their heuristics.

Sanders and Speck [2012] consider the related problem of energy-optimal allocation and mapping of $n$ independent continuously malleable tasks with monotonic and concave speedup functions to $m$ processors with continuous frequency scaling, given a deadline $M$ and a continuous convex energy usage function $E_{j}$ for each task $j$. Continuous malleability means that a fractional number of processors (e.g., using an extra processor only during part of the execution time of a task) can also be allocated for a task. This is not allowed in our task model, in which allocations, speedup, and energy functions are discrete. They propose an almost-linear work algorithm for an optimal solution in the case of unlimited frequency scaling and an approximation algorithm for the case in which frequencies must be chosen between a given minimum and maximum frequency. It is interesting to observe that the assumptions of continuous malleability 
and continuous frequency selection make the integrated problem much easier to solve. Pruhs et al. [2008] solve the problem optimally for sequential tasks with continuous frequencies and power model $f^{\alpha}$.

Related approaches for throughput or energy-efficient (or multi-objective) mapping of complex pipelines have been developed mainly by the MPSoC community for HW/SW synthesis, for example, by using genetic optimization heuristics [Nedjah et al. 2011]. Kessler et al. [2013a] elaborate on the relation between throughput requirements of streaming applications and deadline-based scheduling of independent jobs. They also show that for discrete frequency levels, the optimality criterion of Pruhs et al. [2008] for sequential tasks no longer holds. Keller et al. [2012] and Avdic et al. [2011] have considered mapping of streaming task applications onto processors with fixed frequency with the goal of maximizing the throughput. First investigations of energyefficient frequency scaling in such applications have been done by Cichowski et al. [2012]. Zahedi and Lee [2014] investigate the fair sharing of computer resources to run independent tasks. Fairness is defined after a model derived from game theory to ensure that each task receives enough resources to be computed fast without impacting the performance of other tasks. This work integrates better in a resource-sharing context than in optimizing energy usage of a particular task-based parallel application under makespan constraints, as Crown scheduling does.

Static scheduling of task collections with parallelizable tasks for makespan optimization has been discussed by Blasewicz et al. [2004]. Dynamic scheduling of task collections with parallelizable tasks for flow-time optimization has been investigated by Gupta et al. [2010]. In both settings, frequencies were fixed.

Our LTLG heuristic relates to NPTS (Non-Malleable Parallel Tasks) scheduling, in which the number of cores for each task is known and fixed. Fan et al. [2012] describe previous research, including a 2-approximation [Garey and Graham 1975] and a fully polynomial approximation [Amoura et al. 1997]. More approaches restrict parallel tasks to be mapped to contiguous cores [Baker et al. 1980] such as the NFDH (NextFit Deceasing Height) heuristic [Coffman et al. 1980], FFDH (First-Fit Decreasing Height) [Coffman et al. 1980] and BFDH (Best-Fit Decreasing Height) [Lodi et al. 2002]. However, they do not capture the crown structure that further restricts the mapping of tasks to all cores within exactly one predefined group of cores. The LTLG heuristic is inspired from the LPT (Longest Processing Time) algorithm [Graham 1969], which is a 4/3-approximation algorithm to optimize the makespan of a collection of sequential tasks for multiprocessors.

\section{CONCLUSION AND FUTURE WORK}

We have presented crown scheduling, a new technique for static resource allocation, mapping, and discrete frequency scaling that supports data-driven scheduling of a set of moldable, partly moldable, and sequential streaming tasks onto manycore processors in order to support energy-efficient execution of on-chip pipelined task graphs. Arbitrary power profiles for cores, analytic like $f^{\alpha}$ or from measurements, can be used.

We have presented heuristics and ILP models for the various subproblems and also for an integrated approach that considers all subproblems together, and evaluated these with synthetic benchmarks. Our experimental results show that the complexity reduction imposed by the crown structure constraint, reducing the number of allocatable processor group sizes from $p$ to $O(\log p)$ and of mappable processor groups from $2^{p}$ to $O(p)$, allows for the solution of even medium-sized instances of the integrated optimization problem within a few seconds, using a state-of-the-art ILP solver. The crown structure also minimizes the number of conceptual barrier synchronizations necessary and thus external fragmentation of scheduled idle time, improving opportunities for energy savings by voltage/frequency scaling. 
We presented the Longest Task, Lowest Group (LTLG) heuristic, a generalization of the LPT algorithm, to produce load-balanced mappings of moldable tasks, and the Height frequency scaling heuristic to minimize energy consumption under a throughput constraint. We demonstrated that using these heuristics lowers the overall optimization time of phase-separated crown scheduling, and showed that both LTLG and Height heuristics produce solutions of quality near to that generated by crown-optimal ILP formulations with a much faster scheduling time. We also presented a method based on binary search to compute a good initial allocation and a simulated annealing metaheuristic to further improve solutions toward the quality by an integrated optimal crown scheduler. Our best heuristic runs in near-linear time in the number of cores and tasks with a factor $\gamma$ depending on properties of the task collection. We observe that a good processor allocation improves the energy efficiency of schedules, but that a good allocation comes at a high price in optimization time.

Our technique could be used in a compiler, as we consider off-line scheduling. The inputs would be a description of the task graph with workload and efficiency parameters for tasks (in future work even communication load for edges) and a description of the target architecture. When considering a different target architecture, the application needs to be reoptimized. The crown structure could be relaxed to groups having nonpower-of-2 number of cores, as long as all groups in a level have the same amount of cores and all cores of a higher-level group belong to a unique ancestor group for each lower level. If used to schedule tasks for a multiple manycore chips system, a higher communication penalty to transmit messages from chip to chip, as opposed to communications from core to core, could be reflected in tasks' efficiency function. Future work will also consider online crown rescaling to additionally reduce energy consumption at runtime in a round in which one or several tasks are not data ready, and communication-aware crown scheduling to consider bandwidth requirements of on-chip network links to better adapt to manycore network-on-chip modern architectures.

Also, additional constraints on scaling might be considered, such as power domains comprising whole processor subsets, as in the case of Intel SCC in which possible frequencies are constrained by the current voltage, voltage scaling is slow and can only be set for groups of 8 processors, and frequency scaling is constrained to tiles of two cores. Frequency and sleeping-state switching time need to be taken into account to better model architectural constraints. The energy cost of communication between tasks also needs to be considered in the mapping problem. Finally, we plan to validate energy saving by crown scheduling for a benchmark set on concrete manycore platforms such as the Intel SCC or Kalray MPPA, and compare the performance of our crown scheduler heuristics to other crown and noncrown schedulers for moldable tasks.

\section{REFERENCES}

A. K. Amoura, E. Bampis, C. Kenyon, and Y. Manoussakis. 1997. Scheduling independent multiprocessor tasks. In Algorithms - ESA'97, Rainer Burkard and Gerhard Woeginger (Eds.). Lecture Notes in Computer Science, Vol. 1284. Springer, New York, NY, 1-12. DOI : http://dx.doi.org/10.1007/3-540-63397-9_1

K. Avdic, N. Melot, C. Kessler, and J. Keller. 2011. Parallel sorting on Intel single-chip cloud computer. In Proceedings of the A4MMC Workshop on Applications for Multi-and Many-Core Processors at ISCA 2011.

B. S. Baker, E. G. Coffman, Jr., and R. L. Rivest. 1980. Orthogonal packings in two dimensions. SIAM J. on Computing 9, 4 (Nov. 1980), 846-855.

K. P. Belkhale and P. Banerjee. 1990. An approximate algorithm for the partitionable independent task scheduling problem. In Proceedings of the 1990 International Conference on Parallel Processing. 72-75.

J. Blasewicz, M. Machowiak, J. Weglarz, M. Y. Kovalyov, and D. Trystram. 2004. Scheduling malleable tasks on parallel processors to minimize the makespan: Models and algorithms for planning and scheduling problems. Ann. Oper. Res. 129, 65-80. 
H.-L. Chan, J.f Edmonds, and K. Pruhs. 2011. Speed scaling of processes with arbitrary speedup curves on a multiprocessor. Theory Comput. Syst. 49, 4, 817-833.

P. Cichowski, J. Keller, and C. Kessler. 2012. Modelling power consumption of the Intel SCC. In Proceedings of the 6th MARC Symposium. ONERA, 46-51.

E. Coffman, Jr., M. Garey, D. Johnson, and R. Tarjan. 1980. Performance bounds for level-oriented twodimensional packing algorithms. SIAM J. Comput. 9, 4, 808-826.

J. Edmonds and K. Pruhs. 2012. Scalably scheduling processes with arbitrary speedup curves. ACM Trans. Algorithms 8, 3, 28.

L. Fan, F. Zhang, G. Wang, and Z. Liu. 2012. An effective approximation algorithm for the malleable parallel task scheduling problem. J. Parallel Distrib. Comput. 72, 5, 693-704.

R. Fourer, D. M. Gay, and B. W. Kernighan. 1993. AMPL: A Modeling Language for Mathematical Programming. Scientific Press, South San Francisco.

M. Garey and R. Graham. 1975. Bounds for multiprocessor scheduling with resource constraints. SIAM J. Comput. 4, 2, 187-200. DOI : http://dx.doi.org/10.1137/0204015

M. I. Gordon, W. Thies, and S. Amarasinghe. 2006. Exploiting coarse-grained task, data, and pipeline parallelism in stream programs. In Proceedings of the 12th International Conference on Architectural Support for Programming Languages and Operating Systems (ASPLOS XII). ACM, New York, NY, $151-162$. DOI : http://dx.doi.org/10.1145/1168857.1168877

R. Graham. 1969. Bounds on multiprocessing timing anomalies. SIAM J. Appl. Math. 17, 2, 416-429.

A. Gupta, S. Im, R. Krishnaswamy, B. Moseley, and K. Pruhs. 2010. Scheduling jobs with varying parallelizability to reduce variance. In Proceedings of SPAA'10. ACM, New York, NY, 11-20.

G. Kahn. 1974. The semantics of simple language for parallel programming. In IFIP Congress. 471-475.

J. Keller, C. Kessler, and R. Hulten. 2012. Optimized on-chip-pipelining for memory-intensive computations on multi-core processors with explicit memory hierarchy. J. Universal Comput. Sci. 18, 14, 1987-2023.

C. Kessler, P. Eitschberger, and J. Keller. 2013a. Energy-efficient static scheduling of streaming task collections with malleable tasks. In Proceedings 25th PARS-Workshop (PARS-Mitteilungen). 37-46.

C. Kessler, N. Melot, P. Eitschberger, and J. Keller. 2013b. Crown scheduling: Energy-efficient resource allocation, mapping and discrete frequency scaling for collections of malleable streaming tasks. In Proceedings of the 23rd International Workshop on Power and Timing Modeling, Optimization and Simulation (PATMOS'13).

E. A. Lee and D. G. Messerschmitt. 1987. Synchronous data flow. Proc. IEEE 75, 9, 1235-1245.

K. Li. 2008. Performance analysis of power-aware task scheduling algorithms on multiprocessor computers with dynamic voltage and speed. IEEE Trans. Parallel Distrib. Syst. 19, 11, 1484-1497.

K. Li. 2012. Energy efficient scheduling of parallel tasks on multiprocessor computers. J. Supercomputing 60, 2, 223-247.

A. Lodi, S. Martello, and M. Monaci. 2002. Two-dimensional packing problems: A survey. Eur. J. Oper. Res. 141, 2, 241-252. DOI : http://dx.doi.org/10.1016/S0377-2217(02)00123-6

A. Mazouz, A. Laurent, B. Pradelle, and W. Jalby. 2014. Evaluation of CPU frequency transition latency. Comput. Sci. Res. Dev 29, 3-4 (Aug. 2014), 187-195. DOI : http://dx.doi.org/10.1007/s00450-013-0240-x

N. Nedjah, M. V. Carvalho da Silva, and L. de Macedo Mourelle. 2011. Customized computer-aided application mapping on NoC infrastructure using multiobjective optimization. J. Syst. Arch. 57, 1 (2011), 79-94.

K. Pruhs, R. van Stee, and P. Uthaisombut. 2008. Speed scaling of tasks with precedence constraints. Theory of Computing Systems 43, 1 (July 2008), 67-80.

P. Sanders and J. Speck. 2012. Energy efficient frequency scaling and scheduling for malleable tasks. In Proceedings of the 18th International Conference on Parallel Processing (Euro-Par'12). 167-178.

H. Xu, F. Kong, and Q. Deng. 2012. Energy minimizing for parallel real-time tasks based on level-packing. In Proceedings of the 2012 IEEE 18th International Conference on Embedded and Real-Time Computing Systems and Applications (RTCSA'12). 98-103.

S. M. Zahedi and B. C. Lee. 2014. REF: Resource elasticity fairness with sharing incentives for multiprocessors. SIGARCH Comput. Archit. News 42, 1 (Feb. 2014), 145-160. DOI:http://dx.doi.org/ $10.1145 / 2654822.2541962$

Received May 2014; revised November 2014; accepted November 2014 